\title{
Redo Operation of Recurrent Giant Coronary Artery Aneurysm: Optimizing Surgical Strategy
}

\author{
Julia Hillebrand ${ }^{1} \quad$ Andreas Rukosujew $^{1} \quad$ Sven Marten
}

Dirk Boese ${ }^{2}$
Address for correspondence Dr. Julia Hillebrand, MD, Department of Cardiothoracic Surgery- Division of Cardiac Surgery, University Hospital of the Westfaelische Wilhelms-University Muenster, AlbertSchweitzer-Campus 1, D-48159 Muenster, Germany (e-mail: julia.hillebrand@ukmuenster.de).

\author{
Abstract \\ Keywords \\ - cardiac anatomy \\ - pathologic anatomy \\ - reoperation \\ - cardiovascular \\ surgery
}

Background Giant coronary artery aneurysms (GCAA) are extremely rare, but they can cause life-threatening complications. Indications for surgical intervention are the occurrence of complications such as fistulas, compression, embolization, or rapid enlargement. The optimal therapeutic approach is still under debate.

Until now, there are no publications dealing with the follow-up after surgical treatment. Moreover, the surgical strategy in redo operations has not been described yet.

Case Description We report on surgery in a 50-year-old man with a recurrent GCAA after previous dissection and reduction.

Conclusion Our recommendation is complete resection and bypassing instead of a mere diameter reduction to avoid further progression of the disease.

\section{Introduction}

Coronary artery aneurysms are uncommon and their incidence is described with up to $0.04 \%$ in the general population and up to $4.9 \%$ in patients with the need for coronary angiography. Most often, the right coronary artery is affected (50\%), whereas malformation of the left main coronary artery is observed in only $1 \%{ }^{1}$ A coronary enlargement with a diameter over $2.0 \mathrm{~cm}$ is designated as a giant coronary artery aneurysm, which is an extremely rare pathology, but most likely to be associated with occasionally life-threatening complications. ${ }^{2-4}$

Main causal factors for coronary artery aneurysms are atherosclerosis, congenital disorders, Kawasaki disease or previous coronary artery interventions with or without implantation of drug-eluting stents. Severe complications such as thrombus formation with distal embolization resulting in myocardial ischemia, fistula formation or rupture may occur and the prognosis of untreated coronary artery aneurysms is poor. Several surgical techniques have been described within the last years: thrombectomy, vascular reconstruction as well as coronary artery bypass graft (CABG) with or without aneurysmal ligation. But until now, there is no consensus about the optimal surgical treatment strategy. ${ }^{1-8}$

\section{Case Description}

We report on surgical treatment of a 50-year-old man, who presented with a recurrent giant right coronary artery aneurysm (CAA) and history of cardiac surgery due to a supraaortic stenosis and coronary dilative anomalies especially affecting the right coronary artery.

Primary operative procedure was performed 7 years before and consisted of supracoronary replacement of the ascending aorta and gathering of the right CAA from nearly $5.0 \mathrm{~cm}$ down to approximately $1.0 \mathrm{~cm}$ in diameter after longitudinal dissection.

Within the following years the patient suffered from atrial fibrillation and recurrent progressive coronary dilatation. At the time of presentation the dimension of the CAA showed a re-expansion of approximately $3.0 \mathrm{~cm}$ in diameter and more received

May 13, 2016

accepted after revision

June 17, 2016

published online

September 16, 2016
DOI http://dx.doi.org/

10.1055/s-0036-1586232. ISSN 2194-7635. (c) 2016 Georg Thieme Verlag KG
Stuttgart · New York

License terms

((1) $\Theta \circledast$ 


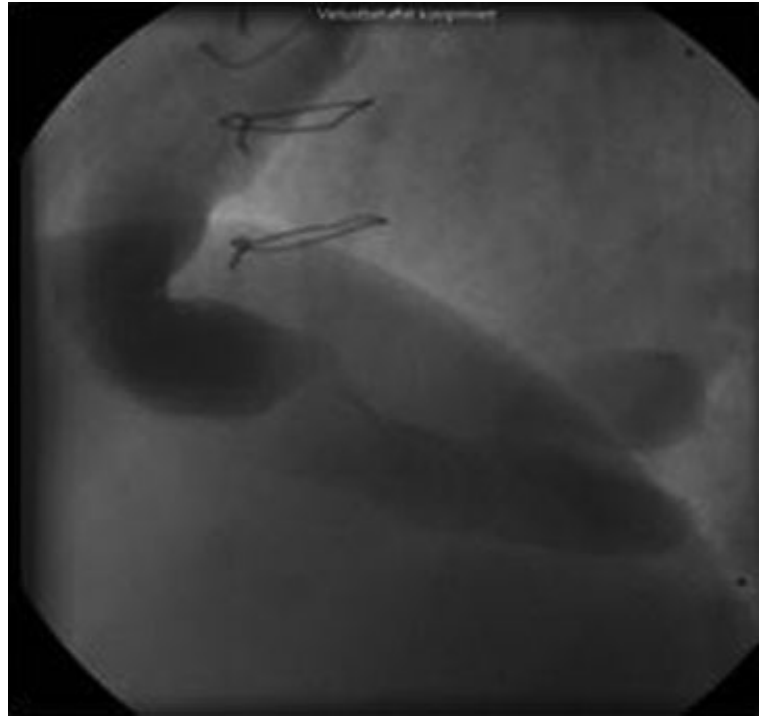

Fig. 1 Coronary angiography (left anterior oblique projection) of the right coronary artery aneurysm with contrast agent.

than $16.0 \mathrm{~cm}$ in length, diagnosed by echocardiography, thoracic computed tomography scan and consecutive coronary angiography. The corresponding preoperative image is depicted in - Fig. 1 and the postoperative angiography in -Fig. 2. Furthermore, the patient developed relevant mitral and tricuspid insufficiency.

Therefore, cardiac surgery was indicated. After installation of extracorporeal circulation via right axillary artery and right femoral vein, we performed median resternotomy. To avoid embolization of atherosclerotic debris or thrombotic material the CAA was carefully dissected after aortic cross clamping. The diameter of the right coronary artery was $8 \mathrm{~mm}$ at the very proximal part and $6 \mathrm{~mm}$ at the crux cordis; acceptable for anastomosis of a venous interponate ( - Figs. 3 and $\mathbf{4}$ ).

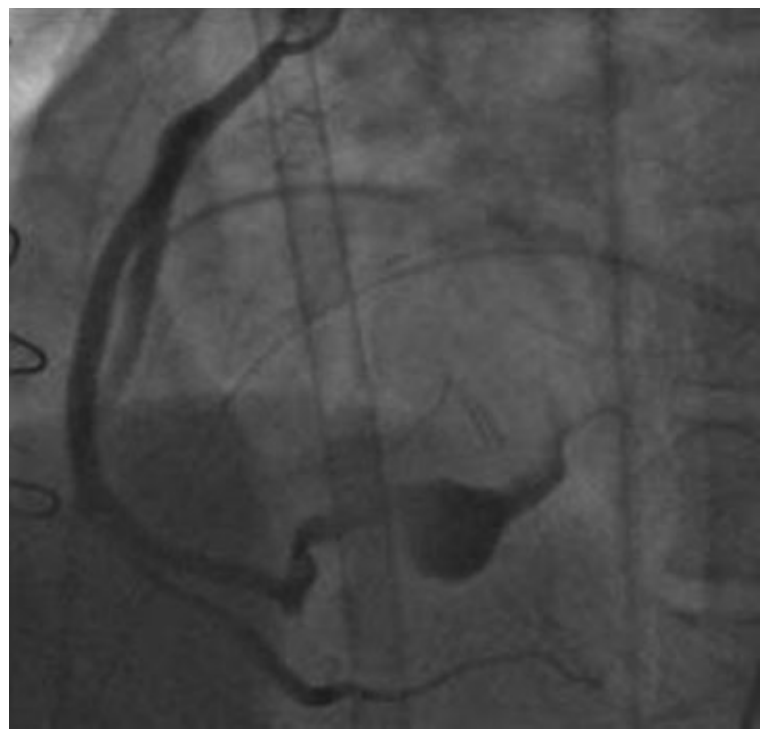

Fig. 2 Postoperative coronary angiography demonstrates sequential bypass grafting for both peripheral branches of the right coronary artery, anastomosed to the venous interponate.

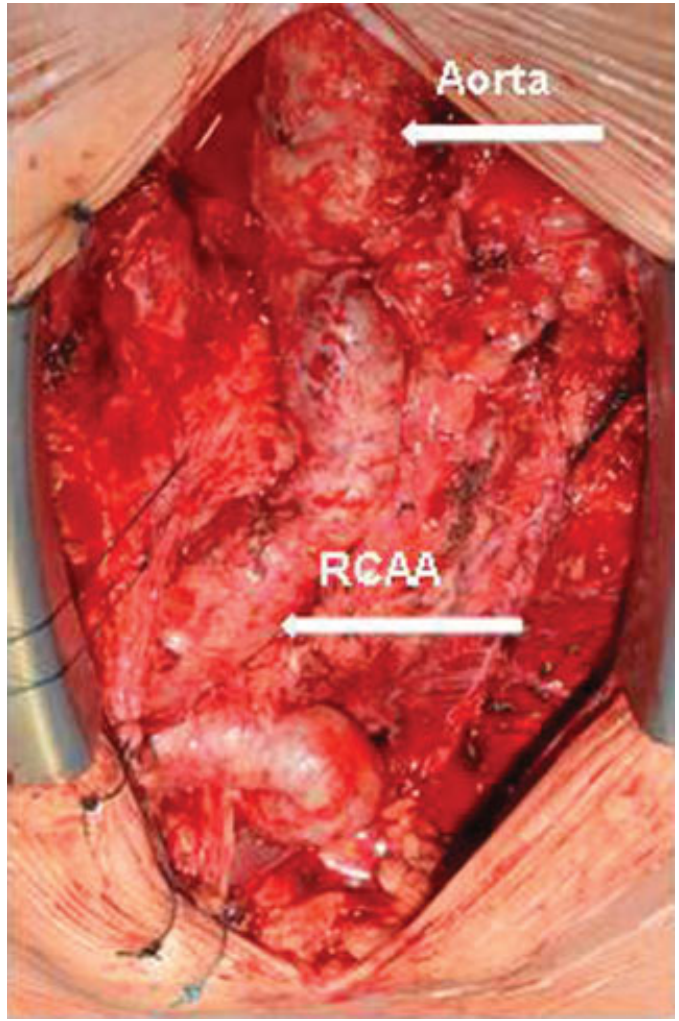

Fig. 3 Redo operative situs. RCAA, right coronary artery aneurysm.

-Figs. 5 and 6 show the extracted CAA with intraluminal thrombus formation. Besides this, the mitral and tricuspid valves were reconstructed, atrial ablation procedure was performed

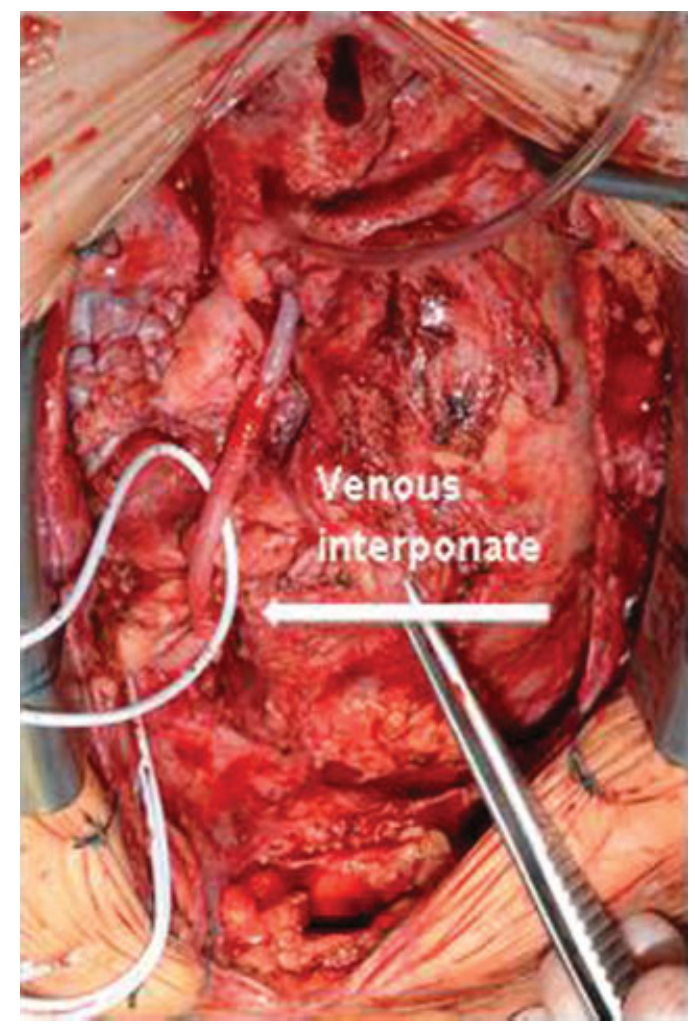

Fig. 4 Venous interponate of the right coronary artery. 


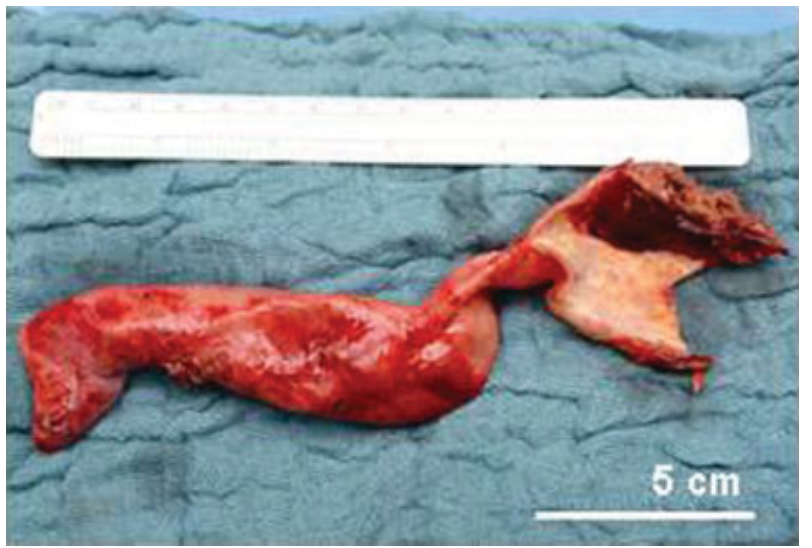

Fig. 5 Intraoperatively excised right coronary artery aneurysm.

and the left appendage was excluded. After weaning from the extracorporeal circulation, we could not achieve sufficient blood flow through the venous interponate, so further revascularization was needed. This was supplied by an additional sequential venous bypass graft to the distal branches of the right coronary artery that was proximally inserted as a Y-graft into the venous interponate (-Fig. 6).

Afterwards weaning from the heart-lung machine was successful and initially hemodynamics was stable. But within the following first postoperative day the patient developed a low cardiac output syndrome. Hence, he required extracorporeal life support and cardiac function recovered within 13 days. The postoperative course was delayed by a transient acute chronic renal failure and a wound infection of the right inguinal. After 71 days, the patient left hospital with good quality of life defined as improved physical capacity (reduction of New York Heart Association class from III to IV down to I to II), possibility of self-supply and freedom of neurologic deficits.

\section{Discussion}

Optimal approach for coronary artery aneurysms is still under discussion. Widely accepted indications for surgical intervention are the occurrence of complications including fistula

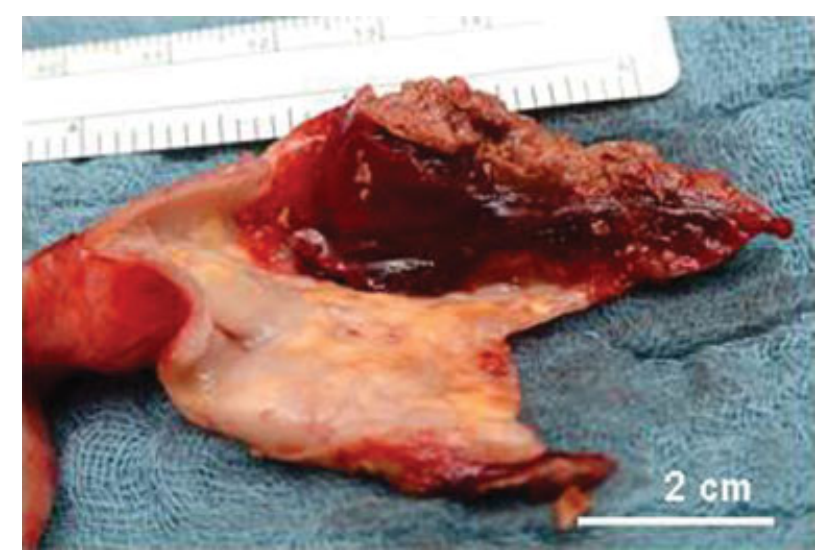

Fig. 6 Image enlargement of the partially dissected right coronary artery aneurysm with thrombotic material. formation, compression, and embolization or rapid enlargement. Particularly in giant coronary artery aneurysms, this technique is thought to be safer and more reliable than other treatment options. ${ }^{6,8}$

In 2013, Singh et al promoted excellent surgical results after proximal ligation, plication, and revascularization. ${ }^{8}$ The presented case demonstrated reoccurrence of relevant enlargement of the right coronary artery aneurysm, 7 years after primary longitudinal dissection and gathering. However, the presented case with a supra-aortic stenosis and this type of recurrence is not the usual case of a coronary aneurysm. Although a connective tissue disorder seems to be likely, none could be detected. We suggest complete aneurysmal resection and bypassing instead of mere diameter reduction as advancing dilation progress can thereby be prevented. Nevertheless, it has to be admitted, that aneurysm resection can be associated with myocardial ischemia and eventually (right) heart failure. Although the thought that eliminating the causal lesion might be beneficial and the superior strategy seems to be self-evident, we can state, that there are hardly any publications dealing with the follow-up and findings after CAA exclusion and even less dealing with the issue of CAA reoperations after previous surgical therapeutic attempt.

According to Holinski et al, we performed CABG to restore coronary perfusion instead of venous interponate alone. ${ }^{3}$

\section{Conclusion}

Until now, treatment strategies for CAAs have not been standardized yet as this vascular malformation is extremely unusual. Individual clinical and anatomical findings should be taken into account for well-grounded therapeutic approach. ${ }^{2}$

\section{References}

1 Genç B, Taştan A, Abacılar AF, Akpınar MB, Uyar S. Thrombosed left circumflex artery aneurysm presenting with myocardial infarction. Asian Cardiovasc Thorac Ann 2016;24(1):39-41

2 Li D, Wu Q Sun L, et al. Surgical treatment of giant coronary artery aneurysm. J Thorac Cardiovasc Surg 2005;130(3):817-821

3 Holinski S, Dohmen PM, Lembcke A, Konertz W. Surgical management of multiple coronary artery aneurysms, including the giant form. Tex Heart Inst J 2009;36(3):238-240

4 Daralammouri Y, Fuhrmann J, Kunze T. Giant right coronary artery aneurysm with a huge intramural thrombus. J Thorac Cardiovasc Surg 2013;146(5):1290-1291

5 Agarwal R, Jeevanandam V, Jolly N. Surgical treatment of a giant coronary artery aneurysm: a modified approach. Ann Thorac Surg 2007;84(4):1392-1394

6 Crawley PD, Mahlow WJ, Huntsinger DR, Afiniwala S, Wortham DC. Giant coronary artery aneurysms: review and update. Tex Heart Inst J 2014;41(6):603-608

7 Ucak A, Inangil G, Selcuk A, Temizkan V. Various surgical approaches to coronary artery aneurysm. J Thorac Cardiovasc Surg 2014;147(4):1434-1435

8 Singh SK, Goyal T, Sethi R, et al. Surgical treatment for coronary artery aneurysm: a single-centre experience. Interact Cardiovasc Thorac Surg 2013;17(4):632-636 